\title{
Alterations in right ventricular pumping in patients with atrial septal defect at rest and during dobutamine stress
}

Sigurdur S Stephensen ${ }^{1,2^{*}}$, Katarina Steding-Ehrenborg ${ }^{2}$, Ulf Thilén ${ }^{3}$, Einar Heiberg ${ }^{2}$, Håkan Arheden ${ }^{2,4}$, Marcus Carlsson 2,4

From 18th Annual SCMR Scientific Sessions

Nice, France. 4-7 February 2015

\section{Background}

CMR can provide details on ventricular pumping by subdividing the contribution to stroke volume (SV) into longitudinal shortening and radial inward motion of the ventricular borders. Previous studies have shown that patients with volume loaded dilated right ventricles (RV) due to pulmonary regurgitation (PR) have decreased longitudinal contribution to RVSV. Patients with atrial septal defects (ASD) also have volume loaded dilated RVs but it is not known if this affects longitudinal shortening.

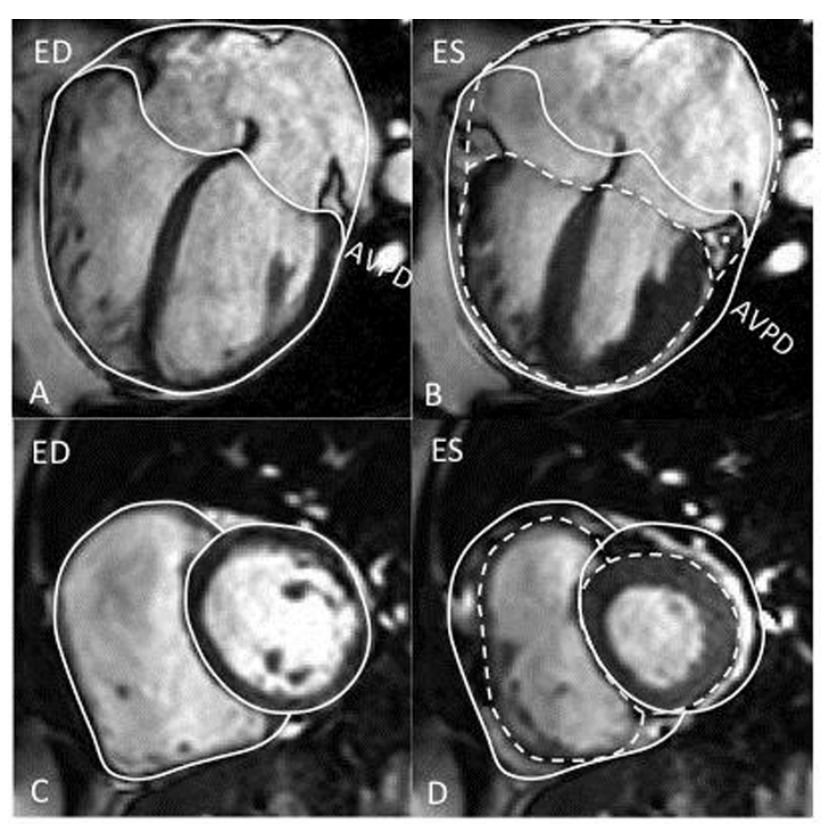

Figure 1 Cardiac MR images in the 4-chamber view (A and B) showing heart volume variations in a patient with ASD. The solid and the dotted white lines represent the heart contours in end-diastole (ED) and end-systole (ES), respectively. Atrioventricular plane displacement (AVPD) caused by longitudinal shortening of the ventricles is marked as a solid white line in ED and a dotted white line in ES.C and D: Short axis images of the same patient. The solid lines represent the epicardial contours of the LV and RV at ED and the dotted lines the epicardial contours at ES. This measurement was done in all short axis slices from the base to the apex. The difference in contours was used to calculate the radial contributions to LVSV and RVSV.

'Department of Pediatric Cardiology, Lund University Hospital, Lund, Sweden

Full list of author information is available at the end of the article 
Table 1 Longitudinal, lateral and septal contribution to stroke volume in patients with atrial septal defect (ASD) and healthy controls at rest and during dobutamine stress.

\begin{tabular}{|c|c|c|c|c|c|}
\hline & $\begin{array}{c}\text { ASD patients preop } \\
\text { at rest }\end{array}$ & $\begin{array}{l}\text { ASD patients during } \\
\text { dobutamine stress }\end{array}$ & $\begin{array}{c}\text { ASD patients postop } \\
\text { at rest }\end{array}$ & $\begin{array}{l}\text { Controls at } \\
\text { rest }\end{array}$ & $\begin{array}{l}\text { Controls during } \\
\text { dobutamine stress }\end{array}$ \\
\hline $\mathrm{HR}(\mathrm{bpm})$ & $73 \pm 11$ & $124 \pm 11^{* * *}$ & $63 \pm 6^{\circ}$ & $65 \pm 10$ & $130 \pm 12^{* * *}$ \\
\hline $\mathrm{CO}(\mathrm{L} / \mathrm{min})$ & $4.8 . \pm 0.9$ & $8.8 \pm 2.8^{* * *}$ & $5.1 \pm 1.2$ & $7.0 \pm 1.5$ & $11.8 \pm 2.1^{* * *}$ \\
\hline $\begin{array}{l}\text { Longitudinal contribution to } \\
\text { LVSV (\%) }\end{array}$ & $63 \pm 12$ & $56 \pm 10$ & $56 \pm 9^{\circ}$ & $61 \pm 7$ & $45 \pm 7^{* * *}$ \\
\hline $\begin{array}{c}\text { Longitudinal contribution to } \\
\text { RVSV }(\%)\end{array}$ & $75 \pm 12$ & $61 \pm 12^{* *}$ & $83 \pm 12^{\circ}$ & $79 \pm 9$ & $57 \pm 10^{* * *}$ \\
\hline $\begin{array}{l}\text { Lateral contribution to LVSV } \\
(\%)\end{array}$ & $39 \pm 12+$ & $46 \pm 13$ & $38 \pm 8$ & $31 \pm 8$ & $46 \pm 7^{* * *}$ \\
\hline $\begin{array}{l}\text { Lateral contribution to RVSV } \\
\qquad(\%)\end{array}$ & $31 \pm 7$ & $38 \pm 7^{*}$ & $27 \pm 10$ & $29 \pm 7$ & $39 \pm 10^{* *}$ \\
\hline $\begin{array}{c}\text { Septal contribution to LVSV } \\
(\%)\end{array}$ & $-2 \pm 13++$ & $-5 \pm 12$ & $6 \pm 6^{\circ}$ & $7 \pm 4$ & $0 \pm 6^{* * *}$ \\
\hline
\end{tabular}

HR, heart rate; CO, cardiac output; LVSV, left ventricular stroke volume; RVSV, right ventricular stroke volume.

${ }^{*} \mathrm{p}<0.05,{ }^{* *} \mathrm{p}<0.01,{ }^{* * *} \mathrm{p}<0.001$ when comparing dobutamine stress to rest for both patients with ASD and healthy controls.

$+p<0.05,++p<0.01$, when comparing patients with ASD preoperatively to healthy controls at rest.

${ }^{\circ} \mathrm{p}<0.05,{ }^{\circ} \mathrm{p}<0.01$, when comparing ASD patients postoperatively and preoperatively.

The purpose of the study was to determine the contribution of longitudinal shortening and radial motion to SV during dobutamine stress and rest in patients with ASD, and to study the early effects of ASD closure on ventricular pumping.

\section{Methods}

Eighteen patients (13 females) with ASD and 16 healthy volunteers (3 females) were imaged with CMR at rest and during a dobutamine/atropine stress protocol. Cine SSFP images were used for LV and RV volumes. Contribution of longitudinal shortening and radial motion to left ventricular (LV) SV and RVSV was measured using manual contouring of short axis images and measurement of longitudinal shortening in three long axis images (Fig 1). The septum was defined as the insertion of the RV into the LV in short axis images. The day following transcutaneous closure of the ASD, a repeated CMR at rest was performed in patients $(n=15)$.

\section{Results}

The left-to-right shunting ratio $(\mathrm{QP} / \mathrm{QS})$ in patients before closure was $2.2 \pm 0.8$. There was no difference in the contribution of longitudinal shortening to SV in patients and controls (Table 1). During dobutaminestress longitudinal shortening contributed less to RVSV and the contribution of radial motion increased. In patients, radial motion of the ventricular septum was towards the RV in systole, contributing to RVSV. This is in contrast to controls where septal motion was towards the LV contributing to LV stroke volume. The day after ASD-closure QP/QS was $1.3 \pm 0.2$ and RV end-diastolic volume (EDV) was decreased compared to before closure $(13 \pm 11 \%)$. Ventricular pumping had changed with a larger contribution of longitudinal shortening to RVSV and radial motion of the septum towards the LV.

\section{Conclusions}

Ventricular pumping in patients with RV dilatation caused by ASD occurs with preserved longitudinal contribution to stroke volume. This differs from previous studies of RV dilatation in patients with pulmonary regurgitation. Dobutamine stress causes similar changes in ventricular pumping in patients with ASD and controls. ASD-closure results in altered ventricular pumping mechanics as early as on day one after treatment.

\section{Funding}

The Swedish Heart-Lung foundation.

\section{Authors' details}

'Department of Pediatric Cardiology, Lund University Hospital, Lund, Sweden. ${ }^{2}$ Cardiac MR group Lund, Dept. of Clinical Physiology, Lund University, Lund, Sweden. ${ }^{3}$ Department of Cardiology, Lund University Hospital, Lund, Sweden. ${ }^{4}$ Dept. of Medical Imaging and Physiology, Lund University Hospital, Lund, Sweden.

Published: 3 February 2015

doi:10.1186/1532-429X-17-S1-Q86

Cite this article as: Stephensen et al:: Alterations in right ventricular pumping in patients with atrial septal defect at rest and during dobutamine stress. Journal of Cardiovascular Magnetic Resonance 201517 (Suppl 1):Q86. 